\title{
Arthroscopic Suture-Button Versus Hook-Plate Fixation for Acromioclavicular Joint Injuries-A Systematic Review of Comparative Studies
}

\author{
Angus J. Lloyd, M.B., B.Ch., Eoghan T. Hurley, M.B., B.Ch., M.Ch., \\ Martin S. Davey, M.B., B.Ch., M.Ch., Leo Pauzenberger, M.D., and \\ Hannan Mullet, M.Ch., F.R.C.S.I. (Tr \& Orth)
}

\begin{abstract}
Purpose: To systematically review the comparative studies in the literature to compare joint stability, clinical outcomes, and complications of acromioclavicular joint fixation using a hook plate versus arthroscopic suture-button (SB) fixation. Methods: A literature search was performed in accordance with the PRISMA guidelines to identify clinical studies comparing the hook plate and arthroscopic SB techniques for acromioclavicular joint injuries. Qualitative statistical analysis was performed using SPSS, and a $P$ value of $\leq .05$ was considered to be statistically significant. Results: Six clinical studies including 285 patients were included in the systematic review. In 3 of the studies, there was a significant difference in favor of SB for Constant score. Patients treated with the SB technique had a lower visual analog scale score at final follow-up in 2 of the 4 studies that measured this outcome. In addition, there were no significant difference in the rate of complications, revisions, or joint malreduction in any of the included studies. Conclusions: The arthroscopic SB procedure resulted in lower postoperative pain scores, and improved postoperative functional outcomes, although this was not a clinically significant difference. In addition, there were no significant differences in the rate of complications, revisions, or joint malreduction. Level of Evidence: Level III, systematic review of Level I, II, and III studies.
\end{abstract}

A cromioclavicular (AC) injuries account for $12 \%$ of all shoulder injuries and $50 \%$ of all sports-related shoulder injuries. ${ }^{1-4}$ The incidence is 5 times greater in men in comparison with women and occurs most commonly in patients 20 to 30 year of age. ${ }^{5}$ Rockwood grade I and II injuries typically are treated conservatively, whereas surgical intervention is commonly required for grade IV-VI injuries. Grade III injuries can be successfully treated with both operative and conservative treatment depending on the individual patient situation. ${ }^{6}$ There are a multitude of procedures

From the Sports Surgery Clinic, Santry (A.J.L., E.T.H., M.S.D., L.P., H.M.); and Department of Trauma $\theta$ Orthopaedic Surgery, Royal College of Surgeons in Ireland (A.J.L., E.T.H., M.S.D.), Dublin, Ireland.

The authors report that they have no conflicts of interest in the authorship and publication of this article. Full ICMJE author disclosure forms are available for this article online, as supplementary material.

Received August 28, 2019; accepted July 3, 2020.

Address correspondence to Eoghan T. Hurley, M.B., B.Ch., M.Ch., Sports Surgery Clinic, Santry, Dublin, Ireland.E-mail:eoghanhurley@rcsi.ie

(C) 2020 THE AUTHORS. Published by Elsevier Inc. on behalf of the Arthroscopy Association of North America. This is an open access article under the CC BY-NC-ND license (http://creativecommons.org/licenses/by-nc-nd/4.0/).

2666-061X/191058

https://doi.org/10.1016/j.asmr.2020.07.005 described in the literature for the surgical management of AC injuries, with no consensus on which surgical technique results in optimal clinical outcomes. ${ }^{7,8}$ Currently the 2 most commonly used techniques are open reduction and fixation with hook-plate (HP) or a suture-button (SB) construct. ${ }^{9-11}$

Fixation using a HP involves the insertion of a precontoured plate that maintains the reduction of the AC joint by acting as a bridge between the lateral aspect of the acromion and the lateral clavicle. ${ }^{12}$ This fixation technique facilitates the natural healing of the joint capsule and the coracoclavicular (CC) ligaments, both of which are involved in stabilizing the AC joint. ${ }^{13}$ It is recommended that the HP is to be removed following adequate healing, typically at 3 to 6 months. ${ }^{12}$

SB fixation allows for a more minimally invasive arthroscopic approach, can be done arthroscopically, and does not require a second surgery to remove the device. ${ }^{14,15}$ Various versions of SB fixation are available varying by surgical technique and manufacturer. Common to all is the use of a pulley system in combination with a point of fixation to stabilize the clavicle and coracoid process. The SB anatomically reduces the distance between the coracoid process and the 
lateral clavicle, allowing the joint capsule and CC ligaments to heal. ${ }^{14}$

Multiple new prospective comparative studies have been published in recent years allowing for an updated systematic review, as previous systematic reviews have consisted entirely of level III or IV evidence, and have included techniques that cannot unreservedly be defined as SB fixation. ${ }^{16,17}$ In addition, previous systematic reviews have contained a mixture of open and arthroscopic techniques. ${ }^{16,17}$ The purpose of this study is to systematically review the comparative studies in the literature to compare joint stability, clinical outcomes, and complications of AC joint fixation using a HP versus arthroscopic SB fixation. Our hypothesis was that the arthroscopic SB fixation would result in decreased postoperative morbidity and improved postoperative outcomes, including increased joint stability, compared with HP fixation.

\section{Methods}

\section{Search Strategy and Study Selection}

Two independent reviewers (A.L. and M.D.) carried out a literature search in accordance with the Preferred Reporting Items for Systematic Reviews and Meta-Analyses guidelines and reviewed the search results. ${ }^{18}$ The following search algorithm: ((ac joint OR acromioclavicular joint OR ac OR acromioclavicular or shoulder) and (sb OR suture button OR tightrope or endobutton or loop or suspensory or arthrosc*) and (hp OR hook plate OR hook plate)) was input into the MEDLINE, EMBASE, and The Cochrane Library databases and all studies were queried from their inception to August 2019. Titles and abstracts were reviewed independently and any study that was potentially eligible underwent a full test review. All articles included in the study were meticulously screened for additional studies within their reference lists that may have met the inclusion criteria.

\section{Eligibility Criteria}

The inclusion criteria included (1) comparative study, (2) comparing the HP and SB techniques, (3) published in a peer-reviewed journal, (4) published in English or full translation freely available, and (5) full-text articles available. The exclusion criteria included (1) nonarthroscopic SB fixation, (2) cadaver studies, (3) review studies, and (4) articles with no full text available or not published in English.

\section{Data Extraction and Quality Assessment}

Two independent reviewers (A.L. and M.D.) extracted the data from the included studies, and these data were confirmed by a third reviewer (E.H.) using a standardized electronic form. Differences of opinion between reviewers was resolved through open discussion and if not resolved the senior author (L.P.) made the final decision. The Oxford Centre for EvidenceBased Medicine criteria were used to assess the level of evidence (LOE). ${ }^{19}$ A Newcastle Ottawa scale was performed on the included studies to further assess study heterogeneity.

\section{Outcomes Analyzed and Statistics}

The outcome measures of interest were the Constant-Murley (CM) score, Taft score, visual analogue scale (VAS) score, coracoclavicular distance (CCD), revisions, joint malreduction (recurrent deformity), and total complications. Qualitative statistical analysis was performed using SPSS, Version 22.0 (Released 2013. IBM SPSS Statistics for Macintosh; IBM Corp., Armonk, NY). A $P$ value of $<.05$ was considered to be statistically significant.

\section{Results}

\section{Literature Search}

The initial literature search resulted in 197 total studies. However, no additional studies were found within the respective reference lists. After removal of duplicates, the articles were screened for inclusion and exclusion criteria, and 114 unique studies were evaluated and full texts were assessed for eligibility. Six clinical studies with 285 patients were included in this review (Fig 1).

\section{Study Characteristics/Patient Demographics}

There were 6 studies (LOE I: 2, LOE II: 1, LOE III: 3) including 135 patients treated with SB and 150 patients treated with HP (Tables 1 and 2). 4,11,13,15,20,21 The median Newcastle Ottawa scale score was 7 (range 6-8). One additional study was removed as it was based on the same randomized controlled trial (RCT) and did had an overlap of patients. The mean age ranged between 30 and 44 years, and the majority of patients were male in all studies (63\%-99\%), with no significant difference in baseline demographics in any study. Six of the studies reported they included only acute AC joint injuries, i.e., $<3$ weeks, and 1 study had a mix of acute and chronic injury. Two studies used doublestrand SB fixation, and 3 studies used single-strand fixation, and one study did not report this. Three studies did not report on HP removal. Of the 3 studies that that did report it, HP removal occurred at 3 months, 6 months, and 12 months. The mean followup ranged between 12 and 48 months.

\section{Constant-Murley Score}

The CM score was reported in 5 studies, with 3 studies finding a significant difference in favor of SB, including the 1 RCT comparing functional 

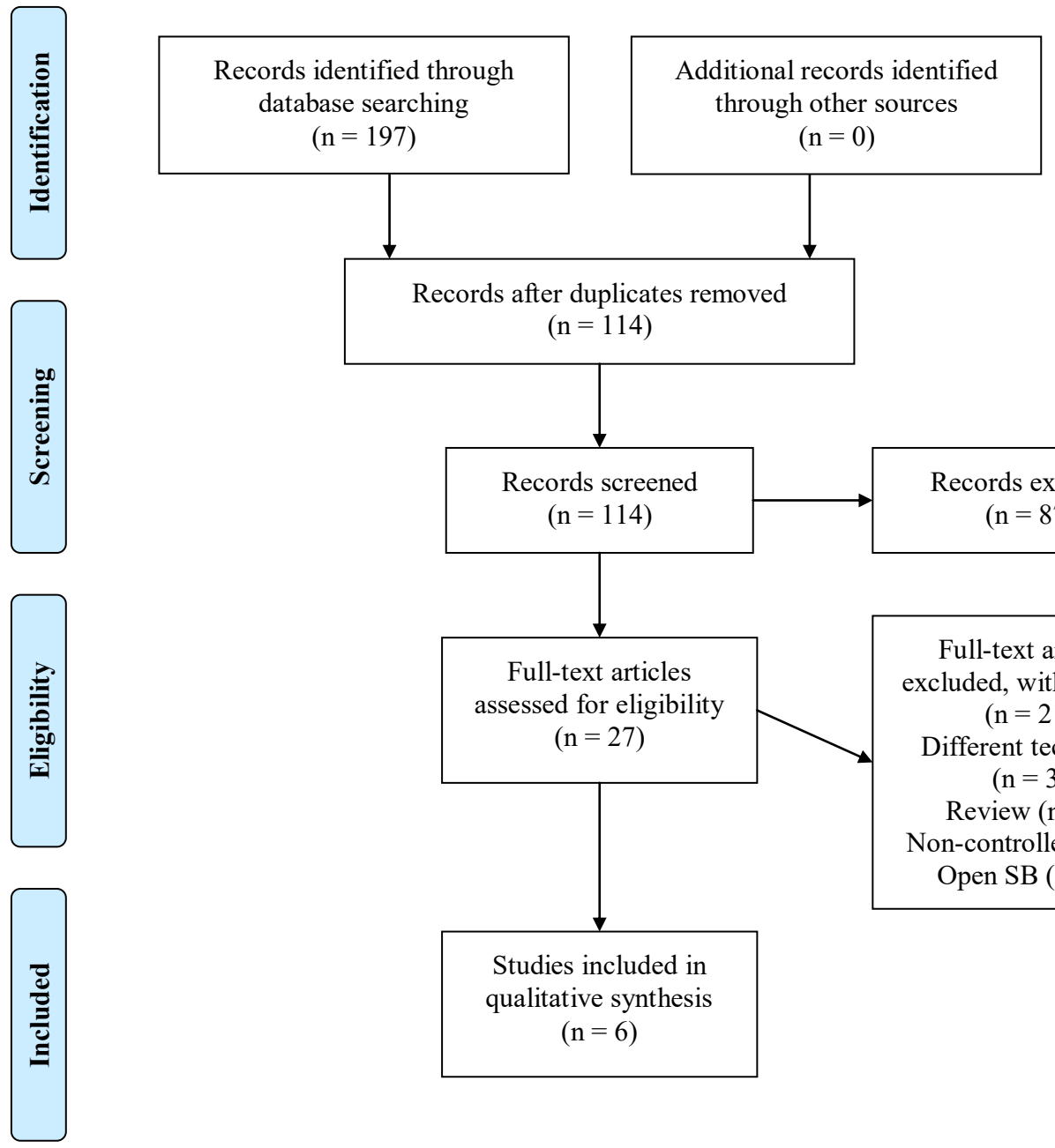

Records after duplicates removed

$(\mathrm{n}=114)$
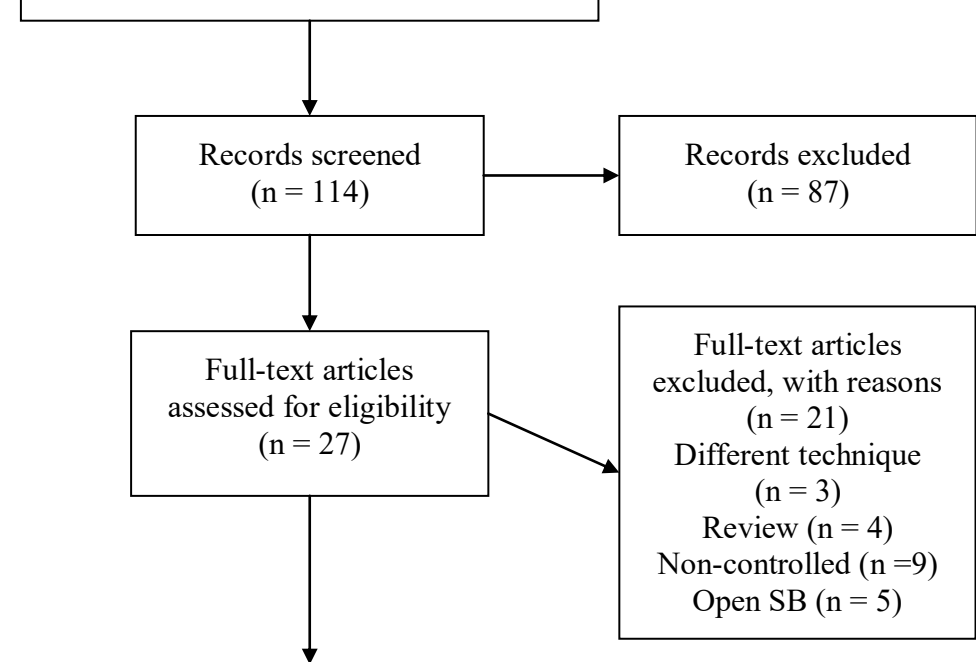

Studies included in

qualitative synthesis $(n=6)$

Fig 1. PRISMA study selection flow diagram (PRISMA, Preferred Reporting Items for Systematic Reviews and Meta-Analyses; SB, suture button.)

outcomes. $^{4,13,15,20,21}$ The mean CM score ranged between 87.6 and 95.3 for SB and between 77.5 and 91.3 for HP.

\section{Taft Score}

The Taft score was reported in 2 studies, with neither study finding a significant difference between the groups, including the 1 RCT comparing evaluating the outcome. The mean Taft score ranged between 10 and 10.7 for SB and 9.4 and 10 for HP.

\section{Visual Analog Scale}

The VAS score was reported in 4 studies, with 2 studies finding a significant difference in favor of SB, including the l RCT comparing evaluating the outcome. The mean VAS score ranged between 0.4 and 1.7 for SB and 1 and 2.4 for HP.

\section{Complications}

Complications were reported in 4 studies, with no study finding a significant difference between SB and

Table 1. Study and Patient Characteristics

\begin{tabular}{|c|c|c|c|c|c|c|c|c|c|}
\hline Author & LOE & Bias & $\mathrm{SB}, \mathrm{n}$ & $\mathrm{HP}, \mathrm{n}$ & Age, y & $\mathrm{M} / \mathrm{F}$ & Grade & Chronicity & $\begin{array}{c}\text { Follow-up, } \\
\text { mo }\end{array}$ \\
\hline Athar et al., $2018^{11}$ & $\mathrm{I}$ & Mod & 24 & 52 & 44 & $75 / 1$ & III/IV & Acute/chronic & 48 \\
\hline Bin Abd Razak et al., $2018^{4}$ & II & High & 16 & 10 & 44 & $24 / 2$ & III-V & Acute & 23 \\
\hline Jensen et al., $2014^{12}$ & III & High & 26 & 30 & 39 & $51 / 5$ & III-V & Acute & 34 \\
\hline Natera-Cisneros et al., $2016^{20}$ & III & High & 20 & 11 & 38 & $28 / 3$ & III-V & Acute & 36 \\
\hline Stein et al., $2018^{13}$ & I & Mod & 29 & 27 & 36 & $54 / 2$ & III-V & Acute & 35 \\
\hline Zhang et al., $2018^{21}$ & III & High & 20 & 20 & 30 & $25 / 15$ & III & Acute & 12 \\
\hline
\end{tabular}

HP, hook plate; LOE, level of evidence; M/F, male/female; mod, moderate; SB, suture button. 
Table 2. Clinical Outcomes

\begin{tabular}{|c|c|c|c|c|c|c|}
\hline & & Outcome Score & $\mathrm{CCD}, \mathrm{mm}$ & Revisions & Malreduction & Complications \\
\hline Author & Outcome Measure & $(\mathrm{SB} / \mathrm{HP})$ & $(\mathrm{SB} / \mathrm{HP})$ & $(\mathrm{SB} / \mathrm{HP})$ & $(\mathrm{SB} / \mathrm{HP})$ & $(\mathrm{SB} / \mathrm{HP})$ \\
\hline Athar et al., $2018^{11}$ & $\mathrm{NA}$ & NA & NA & $31.8 \% / 42.3 \%$ & $25 \% / 3.8 \%$ & $54.2 \% / 32.7 \%$ \\
\hline \multirow{2}{*}{$\begin{array}{l}\text { Bin Abd } \\
\quad \text { Razak et al., } 2018^{4}\end{array}$} & Constant-Murley score & $87.6 / 77.5^{*}$ & $11.8 / 13.6$ & $0 \% / 0 \%$ & NA & $0 \% / 30 \%$ \\
\hline & VAS score & $1 / 1$ & & & & \\
\hline \multirow[t]{3}{*}{ Jensen et al., $2014^{12}$} & Constant-Murley score & $89 / 88$ & $23.8 / 23.0$ & $11.5 \% / 13.3 \%$ & NA & $11.5 \% / 13.3 \%$ \\
\hline & Taft score & $10 / 10$ & & & & \\
\hline & VAS score & $1.3 / 1.7$ & & & & \\
\hline \multirow{2}{*}{$\begin{array}{l}\text { Natera-Cisneros } \\
\text { et al., } 2016^{20}\end{array}$} & Constant-Murley score & $95.3 / 91.3$ & NA & $5 \% / 9.1 \%$ & $40 \% / 36.3 \%$ & $15 \% / 18.2 \%$ \\
\hline & VAS score & $0.4 / 1.5^{*}$ & & & & \\
\hline \multirow[t]{3}{*}{ Stein et al., $2018^{13}$} & Constant-Murley score & $95.3 / 90.1^{*}$ & $16.9 / 19.4$ & NA & NA & NA \\
\hline & Taft score & $10.9 / 9.4^{*}$ & & & & \\
\hline & VAS score & $1.7 / 2.4^{*}$ & & & & \\
\hline Zhang et al., $2018^{21}$ & Constant-Murley score & $95.1 / 85.8^{*}$ & $7.5 / 7.5$ & NA & NA & NA \\
\hline
\end{tabular}

HP. The rate of complications ranged between $0 \%$ and $54.2 \%$ for SB and $13.3 \%$ and $32.7 \%$ for HP. Complications relating to SB were implant failure $(n=1)$, implant sensitivity $(n=2)$, loss of fixation $(n=9)$, acromial erosion $(n=1)$, CC ligament calcification $(n=$ $4)$, and superficial wound infection $(\mathrm{n}=1)$. Complications relating to HP were loss of fixation $(\mathrm{n}=2)$, acromial erosion $(n=2)$, joint stiffness $(n=16)$, acromial fracture $(\mathrm{n}=1)$, AC joint osteoarthritis $(\mathrm{n}=$ $2)$, CC ligament calcification $(\mathrm{n}=4)$, hypertrophic scar $(\mathrm{n}=19)$, and superficial wound infection $(\mathrm{n}=4)$.

\section{Revisions}

Revisions were reported in 4 studies, with no study finding a significant difference between SB and HP. The rate of revisions, excluding routine HP removals, ranged between $0 \%$ and $54.2 \%$ for SB and $13.3 \%$ and $32.7 \%$ for HP.

\section{Joint Malreduction}

Joint malreduction was reported in 2 studies, with neither study finding a significant difference between SB and HP. The rate of joint malreduction between $25 \%$ and $40 \%$ for SB and $3.8 \%$ and $36.3 \%$ for HP.

\section{Coracoclavicular Distance}

The mean CCD was reported in 4 studies, with no study finding a significant difference between SB and HP. The mean CCD ranged between 7.5 and $23.8 \mathrm{~mm}$ for SB and 7.5 and $23 \mathrm{~mm}$ for HP.

\section{Discussion}

The most important finding from this study was that the arthroscopic SB procedure resulted in lower postoperative pain scores and improved postoperative functional outcomes. In addition, there was no significant difference in the rate of complications, revisions, or joint malreduction. However, when including HP routine removals, there was a significantly lower rate of total reoperations with SB.

Our study found there was improved reported functional outcomes between SB and HP, and there was a significant difference in favor of SB fixation for the VAS score. This reduction in pain is most likely a result of the arthroscopic SB technique being less invasive ${ }^{14}$ compared with the HP technique. It has also been noted in the literature that as a result of the HP being fixed to the clavicle on one side and mobile on the other side, patients may experience acromial erosion and damage to the supraspinatus tendon (sub-acromion impingement), causing chronic pain until implant removal. ${ }^{17,22}$ This is supported by Andreani et al., ${ }^{23}$ who reported that $29 \%$ of patients who received the HP complained of pain that dissipated following implant removal. Stein et al. ${ }^{13}$ and Müller et al. ${ }^{24}$ evaluated the rate of return to play following AC joint stabilization and found the arthroscopic SB enabled greater sportspecific outcomes and an ability to return at a greater level. A recent systematic review by Kay et al. ${ }^{25}$ found the rate of return ranged between $94 \%$ and $100 \%$, with rate of return to preinjury level ranging from $62 \%$ to $100 \%$. However, they did not perform any subgroup analysis and did not show a difference between various techniques.

Our study found that there was no statistically significant difference in joint malreduction in the studies that measured this outcome. An intact SB only allows for minimal migration of the clavicle, as Walz et al. ${ }^{26}$ found the SB can withstand greater forces than the native ligaments. However, when it fails or ruptures, then it causes complete malreduction in the joint. In contrast, with the HP it may not completely reduce the clavicle to anatomic levels but due to its increased axial stiffness is less likely to completely malreduce. ${ }^{27}$ In addition, due to the removal of the HP device there may be some minimal migration leading to a greater CCD. ${ }^{15}$ 
No study found a statistically significant difference in complication rate in the studies that measured this outcome. The most prevalent HP complications were implant failure, acromial erosion, and postoperative surficial wound infections. The additional surgery required for implant removal also increases morbidity and the potential risk of infection. Neurologic complications may in theory be more common with HP than SB due to the more-invasive nature of this technique. Fewer complications were associated with SB procedures, and they were mostly related to failure of the implant. Hippensteel et al. ${ }^{28}$ reported that rates of complication, reoperation, and readmission doubled in patients $>40$ years in comparison with patients $<40$ years with AC joint injuries. Complications of the respective surgical techniques should be discussed with patients when counseling them for surgery.

Biomechanical studies have shown the SB technique provides greater stability and stiffness to the joint than the HP in both the superoinferior and anteroposterior planes. Nüchtern et al. ${ }^{27}$ also demonstrated that SB was also superior with respect to internal and external rotational stability. The SB has been reported by Walz et al. ${ }^{26}$ to be able to withstand forces superior than that of native ligaments and provides greater stability to the AC joint, hence facilitating improved healing of the joint capsule and ligaments. Lädermann et al. ${ }^{29}$ reported that the HP does not provide as much anteroposterior support in comparison with the SB, suggesting that $\mathrm{SB}$ provides enhanced stabilisation of the AC joint. In addition, biomechanical studies have found the most common complication seen with HP was a fracture of the lateral clavicle at the most medial screw hole of the hook plate. ${ }^{29}$ As previously reported, SB complications include implant failure resulting in radiologic malreduction of the AC joint, which seems favorable in comparison with an acromial fracture.

\section{Limitations}

The current study has several inherent limitations and potential bias. First, the search criterion was limited to MEDLINE, EMBASE, and Cochrane Library Database articles published exclusively in English. Second, the number of included trials was relatively small, and larger studies may show a significant difference in some results. The included studies were of relatively short follow-up. There was also a moderate risk of bias in some of the included studies; however, this was mostly attributed to the lack of ability to blind assessors and patients due to arthroscopic and non-arthroscopic approach of either technique, as well as the need for routine removal of the HP in many of the studies. Many of the outcome measures being assessed were not reported in all studies. Three of the 6 studies were retrospective in nature. The included studies did not report indications for a specific surgical technique and, therefore, there was a risk of selection bias. In addition, type III injuries may not have required surgical intervention. The possibility of performance bias cannot be ruled out, as surgeons may have had preference for one surgical technique over the other. In addition, the studies with a high risk of bias were deemed high risk due to their non-randomized nature, but the studies did compare comparable baseline demographics in these studies.

\section{Conclusions}

The arthroscopic SB procedure resulted in lower postoperative pain scores and improved postoperative functional outcomes, although this was not a clinically significant difference. In addition, there were no significant differences in the rate of complications, revisions, or joint malreduction.

\section{References}

1. Turnbull JR. Acromioclavicular joint disorders. Med Sci Sports Exerc 1998;30:S26-32.

2. Oussedik S. Injuries to the clavicle and acromioclavicular joint. Br J Hosp Med (Lond) 2007;68:M68-70.

3. Willimon SC, Gaskill TR, Millett PJ. Acromioclavicular joint injuries: Anatomy, diagnosis, and treatment. Phys Sportsmed 2011;39:116-122.

4. Bin Abd Razak HR, Yeo EN, Yeo W, Lie TD. Short-term outcomes of arthroscopic TightRope. Eur J Orthop Surg Traumatol 2018;28:869-875.

5. Mehrberg RD, Lobel SM, Gibson WK. Disorders of the acromioclavicular joint. Phys Med Rehabil Clin North Am 2004; 15:537-555.

6. Rockwood CA, Green DP. Rockwood and Green's fractures in adults. Philadelphia: Lippincott-Raven, 1996.

7. Hoffler CE, Karas SG. Transacromial erosion of a locked subacromial hook plate: Case report and review of literature. J Shoulder Elbow Surg 2010;19:e12-e15.

8. Bannister GC, Wallace WA, Stableforth PG, Hutson MA. The management of acute acromioclavicular dislocation. A randomised prospective controlled trial. J Bone Joint Surg Br 1989;71:848-850.

9. Johansen JA, Grutter PW, McFarland EG, Petersen SA. Acromioclavicular joint injuries: Indications for treatment and treatment options. J Shoulder Elbow Surg 2011;20: S70-82.

10. Simovitch R, Sanders B, Ozbaydar M, Lavery K, Warner JJ. Acromioclavicular joint injuries: Diagnosis and management. J Am Acad Orthop Surg 2009;17:207-219.

11. Athar MS, Ashwood N, Arealis G, Hamlet M, Salt E. Acromioclavicular joint disruptions: A comparison of two surgical approaches 'hook' and 'rope'. J Orthop Surg (Hong Kong) 2018;26:2309499017749984.

12. Kashii M, Inui H, Yamamoto K. Surgical treatment of distal clavicle fractures using the clavicular hook plate. Clin Orthop Relat Res 2006;447:158-164.

13. Stein T, Müller D, Blank M, et al. Stabilization of acute high-grade acromioclavicular joint separation: A prospective assessment of the clavicular hook plate versus the 
double double-button suture procedure. Am J Sports Med 2018;46:2725-2734.

14. Torkaman A, Bagherifard A, Mokhatri T, et al. Doublebutton fixation system for management of acute acromioclavicular joint dislocation. Arch Bone Jt Surg 2016;4: 41-46.

15. Jensen G, Katthagen JC, Alvarado LE, Lill H, Voigt C. Has the arthroscopically assisted reduction of acute AC joint separations with the double tight-rope technique advantages over the clavicular hook plate fixation? Knee Surg Sports Traumatol Arthrosc 2014;22:422-430.

16. Wang C, Meng JH, Zhang YW, Shi MM. Suture button versus hook plate for acute unstable acromioclavicular joint dislocation: A meta-analysis. Am J Sports Med 2019: 363546519858745.

17. Arirachakaran A, Boonard M, Piyapittayanun P, et al. Post-operative outcomes and complications of suspensory loop fixation device versus hook plate in acute unstable acromioclavicular joint dislocation: A systematic review and meta-analysis. J Orthop Traumatol 2017;18:293-304.

18. Liberati A, Altman DG, Tetzlaff J, et al. The PRISMA statement for reporting systematic reviews and metaanalyses of studies that evaluate health care interventions: Explanation and elaboration. PLoS Med 2009; 6:e1000100.

19. Howick JCI, Glasziou P, et al. The Oxford 2011 Levels of Evidence. Oxford Centre for Evidence-Based Medicine. Based Medicine, https://www.cebm.net/index.aspx? $\mathrm{O}=5653$. Accessed September 1, 2019.

20. Natera-Cisneros L, Sarasquete-Reiriz J, Escolà-Benet A, Rodriguez-Miralles J. Acute high-grade acromioclavicular joint injuries treatment: Arthroscopic non-rigid coracoclavicular fixation provides better quality of life outcomes than hook plate ORIF. Orthop Traumatol Surg Res 2016;102:31-39.
21. Zhang L, Zhou X, Qi J, et al. Modified closed-loop doubleendobutton technique for repair of rockwood type III acromioclavicular dislocation. Exp Ther Med 2018;15:940-948.

22. Cai L, Wang T, Lu D, Hu W, Hong J, Chen H. Comparison of the tight rope technique and clavicular hook plate for the treatment of Rockwood type iii acromioclavicular joint dislocation. J Invest Surg 2018;31:226-233.

23. Andreani L, Bonicoli E, Parchi P, Piolanti N, Michele L. Acromio-clavicular repair using two different techniques. Eur J Orthop Surg Traumatol 2014;24:237-242.

24. Müller D, Reinig Y, Hoffmann R, et al. Return to sport after acute acromioclavicular stabilization: A randomized control of double-suture-button system versus clavicular hook plate compared to uninjured shoulder sport athletes. Knee Surg Sports Traumatol Arthrosc 2018;26:3832-3847.

25. Kay J, Memon M, Alolabi B. Return to sport and clinical outcomes after surgical management of acromioclavicular joint dislocation: A systematic review. Arthroscopy 2018;34:2910-2924.

26. Walz L, Salzmann GM, Fabbro T, Eichhorn S, Imhoff AB. The anatomic reconstruction of acromioclavicular joint dislocations using 2 TightRope devices: A biomechanical study. Am J Sports Med 2008;36:2398-2406.

27. Nüchtern JV, Sellenschloh K, Bishop N, et al. Biomechanical evaluation of 3 stabilization methods on acromioclavicular joint dislocations. Am J Sports Med 2013;41: 1387-1394.

28. Hippensteel KJ, Brophy R, Smith M, Wright R. Surgical volume and postoperative complications of acromioclavicular joint separations: Analysis of the ABOS Part II Examination. Am J Sports Med 2018;46:3174-3181.

29. Lädermann A, Gueorguiev B, Stimec B, Fasel J, Rothstock S, Hoffmeyer P. Acromioclavicular joint reconstruction: A comparative biomechanical study of three techniques. J Shoulder Elbow Surg 2013;22:171-178. 